\title{
Efficacy of Tympanoplasty Without Mastoidectomy for Treating Chronic Otitis Media in Patients With Mastoid Cavity Opacification in Temporal Bone Computed Tomography Findings
}

\author{
Hantai $\mathrm{Kim}^{1,2} \cdot$ Ho Young Bae ${ }^{1}$ Oak-Sung Choo ${ }^{1,2} \cdot$ Yun-Hoon Choung ${ }^{1,2,3}$ \\ ${ }^{1}$ Department of Otolaryngology, Ajou University School of Medicine, Suwon; ${ }^{2}$ Department of Medical Sciences, ${ }^{3}$ BK21 Plus Research Center for \\ Biomedical Sciences, Ajou University Graduate School of Medicine, Suwon, Korea
}

Objectives. Combined mastoidectomy is generally preferred to tympanoplasty alone when treating patients with chronic otitis media (COM), particularly when temporal bone computed tomography (TBCT) shows that the mastoid cavity contains opacification of soft tissue density. However, in cases with Eustachian tube dysfunction, a mastoid cavity volume may be a burden to its function. We hypothesized that tympanoplasty alone might be better than tympanoplasty combined with mastoidectomy because soft tissue in the mastoid cavity is a sequel to a protective physiological response. Thus, we explored the efficacy of tympanoplasty without mastoidectomy in COM patients exhibiting mastoid air cell opacification on TBCT.

Methods. Between 2010 and 2014, a total of 33 patients, diagnosed with COM and with evidence of mastoid cavity opacification on TBCT, underwent tympanoplasty without mastoidectomy. All ears had been dry for $\geq 3$ months before surgery. All procedures were performed by the same surgeon. We retrospectively analyzed the preoperative otoscopic findings, pre- and postoperative pure tone averages (PTAs; the mean of the values at $0.5,1,2$, and $4 \mathrm{kHz}$ ), surgical procedures, and complications or recurrence.

Results. Of the 33 patients, 28 (84.8\%) exhibited hearing improvement after surgery. The mean pre- and postoperative PTAs were $46.9 \pm 21.2 \mathrm{~dB}$ and $29.4 \pm 17.0 \mathrm{~dB}$, respectively $(P<0.001)$. The air-bone gap decreased from $25.7 \pm 10.7$ $\mathrm{dB}$ to $10.3 \pm 8.7 \mathrm{~dB}(P<0.001)$. Thirty-two patients $(97.0 \%)$ did not develop any COM recurrence or cholesteatoma; one patient developed attic retraction of the tympanic membrane. Other minor complications were transient otorrhea caused by myringitis (two cases) and a pinpoint perforation (one case).

Conclusion. Tympanoplasty alone, i.e., without mastoidectomy, may adequately control COM, if it shows dry-up status for at least 3 months even though mastoid cavity opacification is detected in TBCT.

Keywords. Otitis Media; Tympanoplasty; Mastoidectomy; Temporal Bone; Computed Tomography

\section{INTRODUCTION}

- Received July 10, 2017

Revised September 10, 2017

Accepted September 23, 2017

- Corresponding author: Yun-Hoon Choung

Department of Otolaryngology, Ajou University School of Medicine,

164 World cup-ro, Yeongtong-gu, Suwon 16499, Korea

Tel: +82-31-219-5263, Fax: +82-31-219-5264

E-mail: yhc@ajou.ac.kr
The efficacy of mastoidectomy combined with tympanoplasty for treating chronic otitis media ( $\mathrm{COM})$ in patients without cholesteatoma remains controversial among otologists. One issue is whether mastoidectomy should be routine when tympanoplasty is performed [1]. Some suggest that mastoidectomy is effective only in patients with suppurative otitis media, and not in those with dry ears [2]. The utility of mastoidectomy is considered un-

Copyright (C) 2018 by Korean Society of Otorhinolaryngology-Head and Neck Surgery.

This is an open-access article distributed under the terms of the Creative Commons Attribution Non-Commercial License (http://creativecommons.org/licenses/by-nc/4.0)

which permits unrestricted non-commercial use, distribution, and reproduction in any medium, provided the original work is properly cited. 
certain, especially in terms of outcomes [3,4].

Today, temporal bone computed tomography (TBCT) plays a key role in COM diagnosis and the choice of surgical intervention $[5,6]$. Air cells of the mastoid cavity and mucosa play important roles in gas exchange, both in the middle ear and mastoid cavity [7]. It may thus be better to preserve, rather than remove, the cavity. Mastoidectomy is unnecessary if the mastoid cavity is clear on TBCT. However, the general view is that mastoidectomy is appropriate when COM is associated with opacification of mastoid cavity.

In terms of physiological recovery, a chronically infected mastoid mucosa will scar during tissue repair [8]. A scar may be physiologically protective, combining with sclerotic bone formation to improve Eustachian tube function. Scar tissue developing in the mastoid cavity after medical and clinical treatment also presents as soft tissue opacification on TBCT. Thus, it may be unwise to remove such tissue via mastoidectomy. We considered that tympanoplasty without mastoidectomy might be a better surgical option for COM patients with soft tissue opacification on TBCT. We thus explored whether tympanoplasty without mastoidectomy was appropriate to treat COM patients in whom TBCT revealed mastoid air cell opacification.

\section{MATERIALS AND METHODS}

A total of 33 ears, of COM patients who had undergone tympanoplasty alone (i.e., without mastoidectomy) and in whom TBCT revealed mastoid cavity opacification, were retrospectively reviewed. All operations were performed by a single surgeon (YHC) at a tertiary referral center (Ajou University Hospital, Suwon, Korea) between 2010 and 2014. Our inclusion criteria were COM without cholesteatoma, no otorrhea for $\geq 3$ months prior to enrolment, and clear middle ear mucosa upon microscopic examination in the operating room. We analyzed preoperative otoscopic findings, pre- and postoperative pure tone averages (PTAs), TBCT images, surgical procedures, and complications or recurrence. Postoperative hearing outcomes were assessed using the relevant Korean guidelines [9]. Surgery was considered successful if the postoperative air-bone gap was $<20$

\section{H I $G$ G H L I I G H T T S}

- Tympanoplasty alone may adequately control chronic otitis media in dry patients.

- Mastoid opacification on computed tomography does not always indicate pathological lesions.

- Tympanoplasty alone successfully improved hearing with a low complication rate.

- A period of medical and clinical management should precede surgery.
$\mathrm{dB}$, the gain in air conduction hearing was $>15 \mathrm{~dB}$, or the postoperative air conduction hearing level was $<30 \mathrm{~dB}$, and when such improvements were assessed by postoperative PTAs performed at least $\geq 6$ months after surgery [9]. This study was performed with the approval of the Institutional Review Board of Ajou University School of Medicine, Korea (IRB No.AJIRB-MEDMDB-17-178). The informed consent was waived.

Soft tissue opacification of mastoid cavity was evaluated by radiologists. Postoperative TBCT was performed if a complication or recurrence was suspected, and to evaluate the opposite side in patients with bilateral COM.

The paired $t$-test was used to assess statistical significance. All statistical analyses were performed with SPSS ver. 18.0 (SPSS Inc., Chicago, IL, USA). A $P$-value $<0.05$ was considered to indicate statistical significance.

\section{RESULTS}

Table 1 shows the baseline characteristics of the study population. The mean patient age was $44 \pm 12$ years and seven ears underwent not only tympanoplasty, but also ossiculoplasty (without mastoidectomy). The mean follow-up period was 19.6 \pm 13.4 months and the minimum period was 6 months.

\section{Hearing results}

Twenty-eight patients (84.8\%) fulfilled at least one of the success criteria described above [9]; five (15.2\%) did not. The mean

Table 1. Patient characteristics

\begin{tabular}{lc}
\hline Characteristic & Value $(\mathrm{n}=33)$ \\
\hline Mean age (yr) & $44 \pm 12$ \\
Sex & \\
Male & $12(36.4)$ \\
Female & $21(63.6)$ \\
Side & \\
Right & $19(57.6)$ \\
Left & $14(42.4)$ \\
Anesthesia & \\
General & $12(36.4)$ \\
Local & $21(63.6)$ \\
Tympanoplasty & \\
Type I tympanoplasty & $26(78.8)$ \\
Type III tympanoplasty & $7(21.2)$ \\
Surgical approach & \\
Transcanal & $22(66.7)$ \\
Retroauricular & $11(33.3)$ \\
Perforation size & \\
Small (<25\%) & $11(33.3)$ \\
Medium (25\%-50\%) & $11(33.3)$ \\
Large (>50\%) & $10(30.3)$ \\
Retraction of the tympanic membrane & $1(3.0)$ \\
Mean follow-up period (mo) & $20 \pm 13$ \\
\hline
\end{tabular}

Values are presented as mean \pm standard deviation or number (\%). 


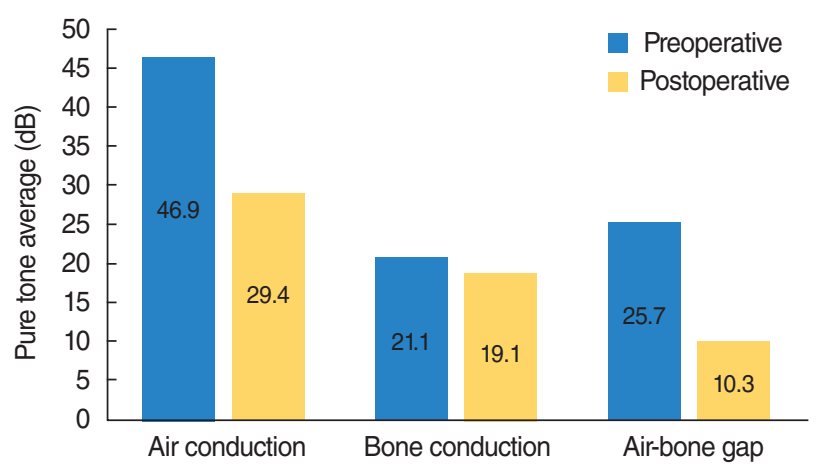

Fig. 1. Audiometric evaluation of the successful 28 patients. Both air conduction and the air-bone gap improved significantly.

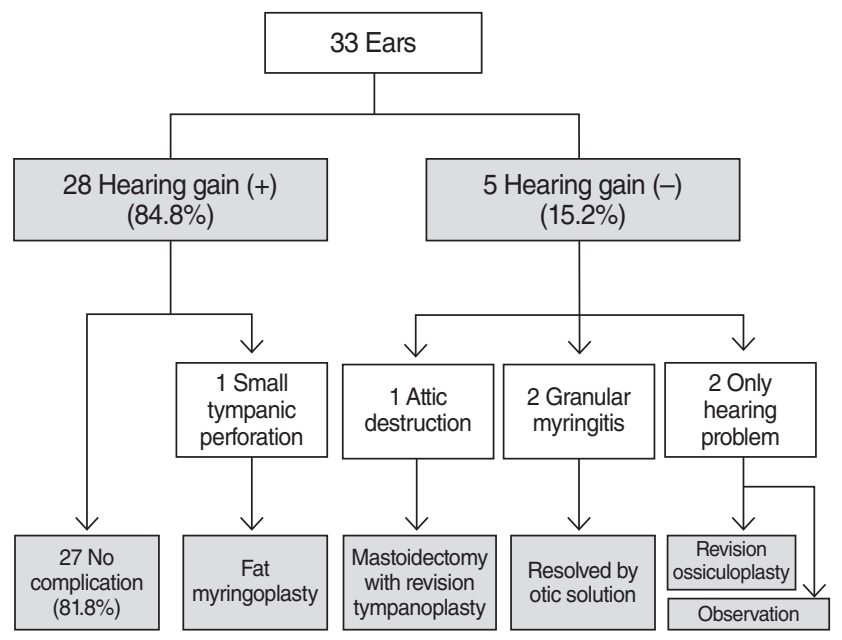

Fig. 2. Complications and their management after tympanoplasty. Mastoidectomy was performed on one patient because of progressive attic destruction after surgery. Overall, $>80 \%$ of patients experienced better hearing outcomes without any complications.

pre- and postoperative PTAs of the 28 successful patients were $46.9 \pm 21.2 \mathrm{~dB}$ and $29.4 \pm 17.0 \mathrm{~dB}$, respectively $(P<0.001)$. The mean pre- and postoperative air-bone gaps of them were $25.7 \pm$ $10.7 \mathrm{~dB}$ and $10.3 \pm 8.7 \mathrm{~dB}$, respectively $(P<0.001)$ (Fig. 1).

\section{Complications and recurrences}

Of the 28 patients exhibiting postoperative hearing gains, one complained of a small tympanic perforation after surgery and underwent fat tissue myringoplasty. Overall, $>80 \%$ of patients enjoyed hearing gains without any complication or recurrence. Attic destruction was developed in one patient during followup; we performed revision tympanoplasty with mastoidectomy. Otoscopic examination revealed otorrhea around tympanic membrane in two patients. These two cases exhibited granular myringitis without a tympanic perforation, which resolved rapidly on application of otic drops. Two patients whose hearing did not improve after tympanoplasty underwent additional ossiculoplasty and further observation (Fig. 2).

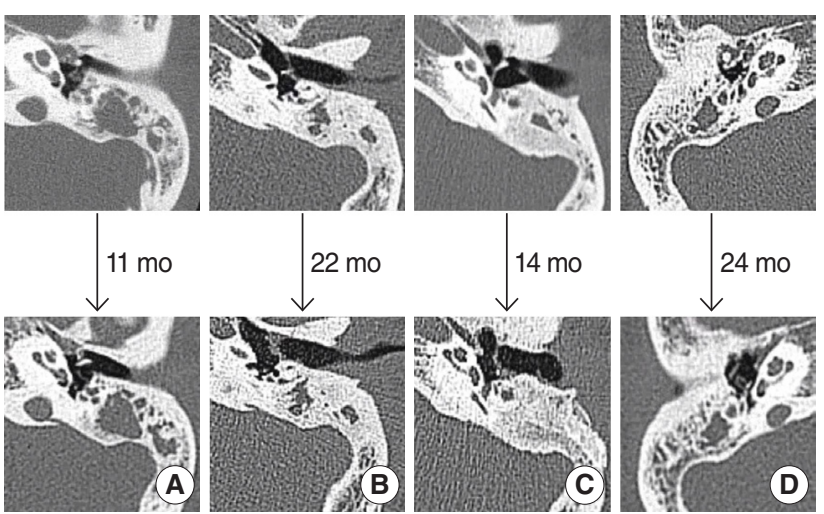

Fig. 3. No change in opacification was evident on follow-up temporal bone computed tomography of four patients. (A) A 22-year-old female: type I tympanoplasty, left. (B) A 47-year-old male: type III tympanoplasty, left. (C) A 42-year-old female: type III tympanoplasty, left. (D) A 52-year-old female: type I tympanoplasty, right.
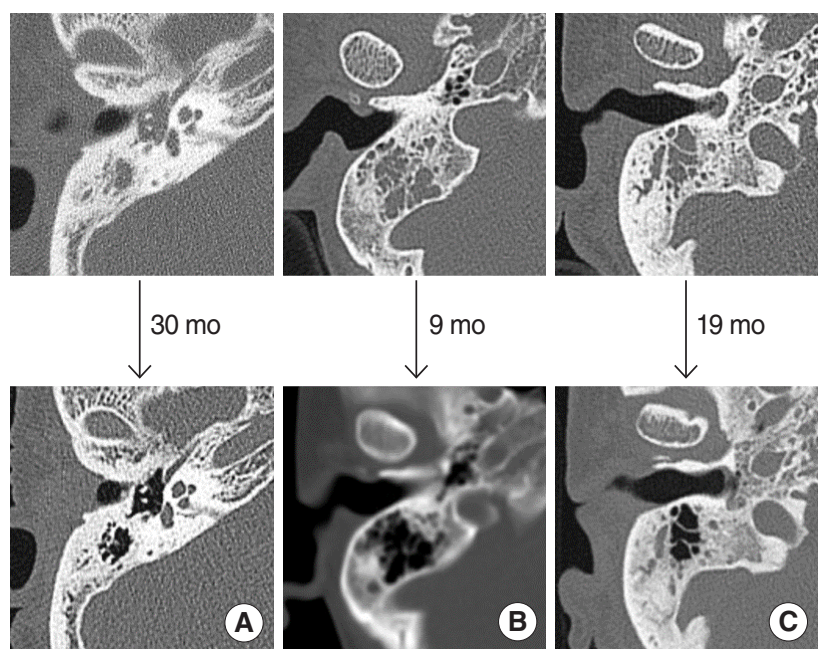

Fig. 4. Resolution of mastoid cavity haziness during post-tympanoplasty follow-up temporal bone computed tomography. (A) A 68year-old male: type I tympanoplasty, right. (B) A 42-year-old female: type I tympanoplasty, right. (C) A 55-year-old female: type I tympanoplasty, right.

\section{Follow-up TBCT}

We performed TBCT after surgeries in seven patients who had ear diseases on the opposite side, complained otorrhea or hearing impairment after surgeries, or were suspicious the possibility of cholesteatoma during follow-up period. In four patients, the preoperative status of the middle ear and mastoid cavity did not change (Fig. 3). The other three patients exhibited no mastoid cavity opacification (Fig. 4).

\section{DISCUSSION}

The middle ear cavity plays an important role in sound conduction, especially by reserving air. There are two principal ear pres- 
sure regulation systems, namely Eustachian tube functionality and gas exchange, principally in the mastoid mucosa $[10,11]$. Takahashi et al. [7] analyzed gas exchange functionality after 14 ear surgeries, showing that it clearly correlated with preservation of the mastoid mucosa. Specifically, mastoidectomized ears lacked gas exchange functionality because the mastoid cavities were filled with thick scar tissue with only a few capillaries [7]. Thus, mastoid cavity preservation is important. However, mastoidectomy is required in certain pathophysiological situations, especially cholesteatoma. This is why great efforts are being made to use tissue engineering to regenerate mastoid cavity air cells [12]. A normal mastoid cavity mucosa is clearly preferable to mastoidectomized or scaffolded regenerated tissue. Therefore, if tympanoplasty alone resolves Eustachian tube obstruction and improves aeration, mastoidectomy is not required.

The significance of mastoid cavity opacification on TBCT requires consideration. In cholesteatoma, for example, opacification reflects a progressive pathological condition that must be eradicated via mastoidectomy. On the other hand, in patients with COM without cholesteatoma, it is doubtful that opacification indicates a pathological lesion that will later trigger disease recurrence. Recently, Wilkinson et al. [13] reported that the prevalence of incidental mastoid cavity and middle ear opacification on TBCT was $11 \%$ in adults without traumatic or otological medical histories.

It has been speculated that mucosal thickening evident on TBCT is a self-limiting process [14]. In terms of tissue repair [8], scars (evident as soft tissue on TBCT) may include normal partregenerated mucosa active in gas exchange; the scar tissue is thus valuable and should not be removed. A mastoidectomized cavity becomes filled with thick scar tissue lacking any gas exchange function [7].

We found that tympanoplasty alone afforded hearing gains in $84.8 \%$ of patients. Attic destruction developed in one patient 11 months after surgery. This indicates that accurate differential diagnosis of COM and cholesteatoma is essential; cholesteatoma patients require mastoidectomy. This was the only case of disease recurrence after operation.

Seven patients underwent follow-up TBCT (Figs. 3, 4). Four cases showed no mastoid cavity changes over time. If the opacification was progressively pathological, adjacent bone destruction would be evident; however, this was not the case, even in the patient with attic destruction (Fig. 3). Fig. 4 illustrates the three cases in whom opacification decreased. We assume that this represented effusion, not scar tissue formation. Effusion is easy to manage on an outpatient basis because no surgery is required.

All 33 cases had undergone medical and clinical management for $\geq 1$ year prior to surgery. Fig. 4 emphasizes the importance of preoperative management (meticulous ear dressing, otic drops, and/or antibiotics). Comprehensive evaluation, including medical history-taking, otoscopic examination, and a period of medical treatment prior to surgery, are critical, as is professional radiological assessment [15].

We suggest that tympanoplasty alone (i.e., without mastoidectomy) when mastoid cavity opacification is evident on TBCT is appropriate when (1) COM is not accompanied by cholesteatoma; (2) the middle ear has been dry for $\geq 1$ year; and (3) sclerotic change is evident in the mastoid cavity. The dry period was $\geq 3$ months in the present study; a longer period would be preferable. Although radiologists seek to differentially diagnose mastoid haziness [15], it is not easy to distinguish between chronic scar tissue and an acute lesion. Our experience (and the findings of Lo and Nemec [15]) suggest that sclerotic change in a retracted mastoid may be key in this context. Clinically, compared to the opposite mastoid, opacification with sclerotic changes indicates mucosal recovery with scar formation, and the mastoid is preserved.

Our study had certain limitations. If our 33 patients had been compared with a group who underwent simple mastoidectomy, the efficacy of tympanoplasty alone would have been clearer. A case-control study is required to confirm our findings. Furthermore, our mean follow-up time was only 19.6 months; longterm follow-up is required.

Tympanoplasty alone may be effective for COM patients even if the mastoid cavity is opaque on TBCT. Before tympanoplasty is performed, the middle ear must be dry. The hearing levels and pre- and perioperative findings must be considered. We suggest that tympanoplasty alone (i.e., without mastoidectomy) is appropriate for patients with $\mathrm{COM}$ without cholesteatoma, whose ears have been dry for $\geq 3$ months and who exhibit sclerotic changes in the mastoid cavity.

\section{CONFLICT OF INTEREST}

No potential conflict of interest relevant to this article was reported.

\section{ACKNOWLEDGMENTS}

This research was supported by the Basic Science Research Program through the National Research Foundation of Korea (NRF) funded by the Ministry of Education, Science and Technology (NRF-2015R1A2A15055956), Republic of Korea.

\section{REFERENCES}

1. Brackmann DE, Shelton C, Arriaga MA. Otologic surgery. 1st ed. Philadelphia:W.B. Saunders; 1994.

2.Vartiainen E, Kansanen M.Tympanomastoidectomy for chronic otitis media without cholesteatoma. Otolaryngol Head Neck Surg. 1992 Mar;106(3):230-4.

3. Mishiro Y, Sakagami M, Kondoh K, Kitahara T, Kakutani C. Long- 
term outcomes after tympanoplasty with and without mastoidectomy for perforated chronic otitis media. Eur Arch Otorhinolaryngol. 2009 Jun;266(6):819-22.

4. Webb BD, Chang CY. Efficacy of tympanoplasty without mastoidectomy for chronic suppurative otitis media. Arch Otolaryngol Head Neck Surg. 2008 Nov;134(11):1155-8.

5. O'Reilly BJ, Chevretton EB, Wylie I, Thakkar C, Butler P, Sathanathan $\mathrm{N}$, et al. The value of CT scanning in chronic suppurative otitis media. J Laryngol Otol. 1991 Dec;105(12):990-4.

6. Leighton SE, Robson AK, Anslow P, Milford CA. The role of CT imaging in the management of chronic suppurative otitis media. Clin Otolaryngol Allied Sci. 1993 Feb;18(1):23-9.

7. Takahashi H, Honjo I, Naito Y, Miura M, Tanabe M, Hasebe S, et al. Gas exchange function through the mastoid mucosa in ears after surgery. Laryngoscope. 1997 Aug;107(8):1117-21.

8. Kumar V, Abbas AK, Aster JC. Robbins and Cotran pathologic basis of disease. 9th ed. Philadelphia: Elsevier Saunders; 2015.

9. Kim HJ. Classification and hearing result reporting guideline in chronic otitis media surgery. Korean J Otorhinolaryngol Head Neck
Surg. 2006 Jan;49(1):2-6.

10. Hergils L, Magnuson B. Morning pressure in the middle ear. Arch Otolaryngol. 1985 Feb;111(2):86-9.

11. Buckingham RA, Stuart DR, Geick MR, Girgis SJ, McGeeTJ. Experimental evidence against middle ear oxygen absorption. Laryngoscope. 1985 Apr;95(4):437-42.

12. Kanemaru S, Nakamura T, Omori K, Magrufov A, Yamashita M, Ito J. Regeneration of mastoid air cells in clinical applications by in situ tissue engineering. Laryngoscope. 2005 Feb;115(2):253-8.

13. Wilkinson SL, Sahota RS, Constable JD, Harper F, Judd O. Does incidental mastoid opacification on computerized tomography necessitate referral to ENT? Laryngoscope. 2017 Dec;127(12):2860-5.

14. Gwaltney JM Jr, Phillips CD, Miller RD, Riker DK. Computed tomographic study of the common cold. N Engl J Med. 1994 Jan;330 (1):25-30.

15. Lo AC, Nemec SF. Opacification of the middle ear and mastoid: imaging findings and clues to differential diagnosis. Clin Radiol. 2015 May;70(5):e1-13. 